\title{
Joint sun-glitter and radar imagery of surface slicks
}

\author{
Vladimir Kudryavtsev a, b, d, Alexander Myasoedov ${ }^{a, b, ~}{ }^{\text {, }}$, Bertrand Chapron ${ }^{c}$, Johnny A. Johannessen $^{\mathrm{d}, \mathrm{e}}$, \\ Fabrice Collard
}

\author{
a Nansen International Environmental and Remote Sensing Center, St. Petersburg, Russia \\ ${ }^{\mathrm{b}}$ Russian State Hydrometeorological University, St. Petersburg, Russia \\ c Institute Francais de Recherche pour l'Exploitation de la Mer, Plouzané, France \\ ${ }^{\mathrm{d}}$ Nansen Environmental and Remote Sensing Center, Bergen, Norway \\ ${ }^{\mathrm{e}}$ Geophysical Institute, University of Bergen, Norway \\ ${ }^{f}$ CLS-Direction of Radar Applications, Plouzané, France \\ *: Corresponding author : Alexander Myasoedov, email address : alexander.myasoedov@gmail.com
}

\begin{abstract}
:
A method is proposed to retrieve and interpret fine spatial variations of the sea surface roughness in sun glitter imagery. Observed sun glitter brightness anomalies are converted using a transfer function determined from the smoothed shape of sun glitter brightness. The method is applied to MODIS and MERIS sun glitter imagery of natural oil seeps and the catastrophic Deepwater Horizon oil spill in the Gulf of Mexico. The short-scale roughness variations in the presence of mineral oils slicks are consistently extracted and compared to variations associated with the biogenic slicks. In doing so, the wind speed dependency on the roughness anomalies is also considered. A comparison to normalized radar cross section (NRCS) anomalies taken from the corresponding high resolution ASAR images is performed, and similarities as well as differences are investigated. The results document significant benefit from the synergetic use of sun glitter and radar imagery for detection and monitoring of surface slicks.
\end{abstract}

\section{Highlights}

Estimation of the spatial anomalies in the mean square slope of the sea surface. Distinct relationship between sun-glitter and radar backscatter contrasts. Collocated MODIS, MERIS and ASAR images. Film elasticity coefficient. Consistent optical and radar imaging model.

Keywords : Sun-glitter ; Mean square slope ; Surface slicks ; Oil spills ; SAR imaging model ; SAR and optical synergy 


\section{Introduction}

29 In general, the main oceanographic applications of satellite optical data (e.g. from

30 MODIS and MERIS instruments) are associated with ocean color studies. In such cases

31 the sunlight reflected from the sea surface is a major part of upward radiation and possess

32 significant difficulties for ocean color retrieval algorithms. On the other hand, sun glitter

33 contain valuable information on statistical properties of the sea surface roughness, its

34 mean square slope (MSS), skewness and kurtosis, as demonstrated by Cox and Munk

35 (1954) and more recently by Bréon and Henriot (2006).

36 Most ocean surface phenomena, e.g. biogenic and oil slicks, internal waves, ship

37 wakes, spiral eddies, are locally affecting sea surface roughness to become visible in

38 optical data. Numerous satellite observations of surface slicks in sun glitter were

39 reported, e.g. by Adamo et al. (2005), Chust and Sagarminaga (2007), and Hu et al.

40 (2009). Hennings et al. (1994) presented observations of the surface manifestation of

41 shallow water bottom topography in sun glitter brightness. Apel et al. (1975), Artale et al.

42 (1990), and Mitnik et al. (2000) observed and studied non-linear internal waves in sun

43 glitter imagery. Jackson (2007) used MODIS sun glint imagery to determine the spatial

44 distribution of internal waves (IWs) over the global ocean.

45 Evidently, sun glitter signatures are caused by spatial variation of short scale sea

46 surface roughness tracing surface manifestation of an ocean phenomenon. The magnitude

47 of the contrasts is connected to the type of surface slick, e.g. biogenic, oil, and possibly

48 thickness of the oil spill producing the slick. Retrieval and quantitative interpretation of

49 these sun glitter brightness contrasts can thus help to better understand damping

50 mechanisms. 
There are few papers focused on the specific problem of retrieval of quantitative

52 roughness anomalies from high resolution sun glitter imagery. Burdyugov et al. (1987)

53 used the Cox and Munk (1954) model to convert sun glitter brightness signatures into

54 MSS contrasts and applied their approach to airborne photographs of surface slicks

55 produced by a train of IWs. Reconstruction of 2D spectra of dominant surface wave

56 elevations from sun glitter have also been reported (e.g., Stillwell, 1969; Bolshakov et

57 al., 1990a) and used to investigate the evolution and transformation of 2D wind wave

58 spectra (Bolshakov et al. 1990b).

59 Unlike determination of the background statistical properties of the sea surface

60 slopes, to the best of our knowledge, satellite sun glitter imagery has never been

61 specifically used for quantitative estimates of MSS anomalies of the surface roughness.

62 Complexity arises from the fact that the sun glitter brightness and the MSS contrasts

63 depend on the viewing and sun illumination geometries. Contrasts of MSS can then

64 either be visible as dark/bright or bright/dark brightness signatures (see e.g. Hu et al.

65 (2009) for the oil slicks; Matthews (2005), Munk et al. (1987) for ship wakes and IWs;

66 Munk et al. (2000) for spiral eddies; Jackson and Alpers (2010) for IWs and oil slicks).

67 This property is quite straightforward, and as shown by Burdyugov et al. (1987) and

68 recently by Jackson and Alpers (2010), is a simple consequence of different viewing

69 distance angles from the specular point.

70 The goal of this study is to develop a method to quantitatively and consistently

71 retrieve MSS anomalies from brightness signatures under various viewing geometries

72 coincidentally with radar backscatter damping in SAR images. The study is mainly

73 focused on M ODIS, MERIS and ASAR observations, though application of the

74 suggested method for interpretation of sun glitter images received from other optical 
75 sensors is straightforward. Examples of the application of the suggested method are

76 given for surface roughness variations in presence of oil slicks.

\section{2. Retrieval of the MSS anomalies}

78 Satellite optical images collected during the daylight period contain distinct silvery-

79 gray ellipses of reflected sunlight over the oceans within approximately 30 degree of the

80 Sun's specular reflection point. These sun glitter regions, where standard ocean color

81 products cannot usually be retrieved, can be more favorable for detecting damping

82 mechanisms for surface roughness. To sense roughness changes, the red channel is the

83 most preferable one, as the light in this channel is absorbed within a "thin" surface layer

84 and, thus, is not too sensitive to the optical properties of the upper water column.

85 Moreover, it does not depend on s ea surface temperature. Considering MODIS and

86 MERIS imagery, we use the Level 1B $250 \mathrm{~m}$ resolution data in $645 \mathrm{~nm}$ channel for

87 MODIS and $681 \mathrm{~nm}$ channel for MERIS which are supplemented with geolocation and

88 "view and sun geometry" data.

\subsection{Relation of brightness and MSS anomalies}

90 We consider the surface brightness field in the sun glitter area where the impact of

91 the sky radiance reflected from the surface to the sensor is negligible. Following Cox and

92 Munk (1954) the sun glitter radiance, $B$, generated by specular reflection of the sun light

93 is given as

94

$$
B=\frac{\rho E_{s}}{4 \cos \theta_{v} \cos ^{4} \beta} P\left(Z_{x}, Z_{y}\right),
$$

95 where $E_{s}$ is the solar irradiance, $\rho$ is the Fresnel reflection coefficient, $\theta_{v}$ is the view

96 zenith angle, $P$ is the 2D probability density function (PDF) of the sea surface slopes $z_{x}$ 
97 and $z_{y}$, and capital $Z_{x}$ and $Z_{y}$ denote the sea surface slopes and their values satisfying

98 the conditions of specular reflections of the sun light received by the sensor

99

$$
\begin{aligned}
& Z_{x}=-\frac{\sin \theta_{s} \cos \varphi_{s}+\sin \theta_{v} \cos \varphi_{v}}{\cos \theta_{s}+\cos \theta_{v}} \\
& Z_{y}=-\frac{\sin \theta_{s} \sin \varphi_{s}+\sin \theta_{v} \sin \varphi_{v}}{\cos \theta_{s}+\cos \theta_{v}}
\end{aligned}
$$

100 where $\theta_{s}$ is the sun zenith angle, $\varphi_{v}$ and $\varphi_{s}$ are the view and sun azimuth angles, and $101 \tan \beta=\sqrt{Z_{x}^{2}+Z_{y}^{2}}$. Cox and Munk (1954) and, later, e.g. Chapron et al. (2000) and Bréon 102 and Henriot (2006), suggested to model the 2D sea surface PDF as non-Gaussian, taking 103 into account the non-linearity of the surface wave slopes.

104 Additional opportunities exist for high-resolution satellites with large fields of view 105 to investigate sea surface phenomena that lead to variations of the sea surface 106 "roughness". The brightness field, $B$, can indeed be decomposed into a large-scale 107 background part $B_{0}$ and a s mall-scale detail part $\tilde{B}: B=B_{0}+\tilde{B}$. Field $B_{0}$ can 108 correspond to the brightness at the scale of the sun glitter width, $L$ (order of hundred $109 \mathrm{~km}$ ), while field $\tilde{B}$ contains brightness details at much smaller scales, $l \ll L$, (order of 110 ten $\mathrm{km}$ ), which can be treated as the brightness signatures of the ocean phenomena. Let 111 the PDF, $P$, in (1) be written in a normalized form as

$$
P\left(Z_{x}, Z_{y}\right)=s^{-2} p(\xi, \eta)
$$

113 where $\xi=Z_{x} / s$, and $\eta=Z_{y} / s, s^{2}$ is the mean squared slope (MSS) of the sea surface, 114 and $p$ is a "scaled" PDF. Variations of $s^{2}$ (as well as other statistical characteristics of 
115 the surface slopes) can then be represented as a s um of a m ean value, $s_{0}^{2}$, and its

116 variations, $\tilde{s}^{2}$

$$
s^{2}=s_{0}^{2}+\tilde{s}^{2}
$$

118 As assumed, variations $\tilde{s}^{2}$ take place on the small inner scale $l$, while $s_{0}^{2}$ may be 119 related to the background sun glitter width scale $L$.

120 The MSS is mostly supported by wind waves shorter than $\mathrm{O}(1) \mathrm{m}$ (Cox and Munk, 121 1957; Vandemark et al., 2004), thus variations of the MSS can be expected to trace local

122 features of the ocean phenomena. Spatial variations of MSS with small $\tilde{s}^{2} / s_{0}^{2}$, in 123 equations (1) and (3), will give the following sun glitter brightness variations

$$
\ln \left(\frac{B_{0}+\tilde{B}}{B_{0}}\right)=-T \frac{\tilde{s}}{s_{0}^{2}}
$$

125 where $T$ is the transfer function defined as

$$
T=1+\frac{1}{2}\left(\frac{\partial \ln p}{\partial \ln \xi}+\frac{\partial \ln p}{\partial \ln \eta}\right)
$$

127 For sake of simplicity, to derive (5), we assumed that $\tilde{s}^{2}$ dominates and controls 128 variations of other statistical parameters of the surface slopes, in particular the 129 directionality, peakedness and skewness. In other words, it is assumed that the magnitude 130 of the relative MSS variations $\tilde{s}^{2} / s_{0}^{2}$ is significantly larger than variations of other sea 131 slope statistical moments $\overline{z_{x}^{m} z_{y}^{n}}$ scaled by the MSS, $c_{m n}=\overline{z_{x}^{m} z_{y}^{n}} / s^{m+n}$, i.e. $132 s^{2} / s^{2} \gg \tilde{y}_{m n} / c_{m n}$. This assumption is supported by measurements of the MSS of clean 133 and slick covered surface by Cox and Munk (1954). According to their measurements,

134 the ratio between clean and slick covered areas is $\left(s^{2}\right)_{\text {clean }} /\left(s^{2}\right)_{\text {slick }} \approx 1.5-2$ for moderate 
135 wind conditions. At the same time the normalized up-wind $c_{20}$ and cross-wind $c_{02}$ slope 136 anisotropy parameters vary insignificantly, i.e. $\left(c_{20}\right)_{\text {clean }} /\left(c_{20}\right)_{\text {slick }} \approx 1 \pm 0.1$ and $137\left(c_{02}\right)_{\text {clean }} /\left(c_{02}\right)_{\text {slick }} \approx 1 \pm 0.1$. While the MSS is strongly suppressed in the slick areas, 138 coefficients of the slope anisotropy over the slick do not show significant changes. The 139 transfer function $T$ defined by $(5 b)$ can then be found either empirically, using 140 "measured" gradients of the sun glitter brightness, or theoretically if the PDF has a 141 predefined form.

142 To illustrate our purpose, we can first consider the 2D Gaussian PDF. In this case the 143 scaled PDF $p \equiv s^{2} P$ is

$$
p\left(Z_{x}, Z_{y}\right)=\frac{s^{2}}{2 \pi s_{u} s_{c}} \exp \left[-\frac{s_{y}^{2} Z_{x}^{2}-2 \mathrm{~s}_{x y}^{2} Z_{x} Z_{y}+s_{x}^{2} Z_{y}^{2}}{2 s_{u}^{2} s_{c}^{2}}\right]
$$

145 where $(x, y)$ is an arbitrary orthogonal coordinate system, and $s_{x}^{2}$ and $s_{y}^{2}$ are 146 components of the MSS in this coordinate system related to the up- and cross-wind MSS

147 components ( $s_{u}^{2}$ and $s_{c}^{2}$ correspondingly) defined as

$$
\begin{aligned}
s_{x}^{2} & =s_{u}^{2} \cos ^{2} \varphi+s_{c}^{2} \sin ^{2} \varphi \\
s_{y}^{2} & =s_{c}^{2} \cos ^{2} \varphi+s_{u}^{2} \sin ^{2} \varphi \\
s_{x y}^{2} & =\left(s_{u}^{2}-s_{c}^{2}\right) \cos \varphi \sin \varphi
\end{aligned},
$$

149 where $\varphi$ is wind direction. Let $\alpha=s_{c}^{2} / s_{u}^{2}$ be a parameter of the MSS slope anisotropy.

150 Then, the dimensionless PDF (7) can be rewritten as

$$
p(\xi, \eta)=\frac{1+\alpha}{2 \pi \alpha^{1 / 2}} \exp \left[-a_{2} \xi^{2}+a_{12} \xi \eta-a_{1} \eta^{2}\right],
$$

152 where coefficients $a_{1}, a_{2}$ and $a_{12}$ are 


$$
\begin{aligned}
& a_{1}=(1+\alpha)\left(\cos ^{2} \varphi+\alpha \sin ^{2} \varphi\right) /(2 \alpha) \\
& a_{2}=(1+\alpha)\left(\alpha \cos ^{2} \varphi+\sin ^{2} \varphi\right) /(2 \alpha) \\
& a_{12}=\left(1-\alpha^{2}\right) \sin 2 \varphi /(2 \alpha)
\end{aligned}
$$

154 For the isotropic slope PDF, $\alpha=s_{c}^{2} / s_{u}^{2}=1$, the coefficients reduce to $a_{1}=a_{2}=1$ and $155 a_{12}=0$.

156 As discussed above, we can assume that the anisotropy coefficient $\alpha$ does not vary, 157 and the transfer function becomes

$$
T=1-\left(a_{2} Z_{x}^{2}-a_{12} Z_{x} Z_{y}+a_{1} Z_{y}^{2}\right) / s_{0}^{2}
$$

159 Accordingly, to retrieve the MSS anomalies, one needs to know the wind direction,

$160 \varphi$, the background roughness, $s_{0}^{2}$, and the anisotropy coefficient, $\alpha$. In an "ideal" case,

161 the parameters $s_{0}^{2}, \alpha$, and $\varphi$ can be determined by fitting the mean 2D brightness field 162 (Bréon and Henriot, 2006). Unfortunately, satellite scanners, e.g. MODIS and MERIS, 163 mostly provide observations in one cross-track direction, and the proper 2D information 164 of the brightness field is not available. In such a case, the wind direction cannot be 165 derived, and must be considered as an "outer" parameter which could be introduced from 166 other data sources, e.g. from meteorological data. Yet, following observations from Cox 167 and Munk (1954) or Bréon and Henriot (2006), the anisotropy coefficient $\alpha$ does not 168 significantly vary with wind speed. For practical applications, a mean value $\alpha=0.7$ can 169 be chosen, and $s_{0}^{2}$ can be robustly derived from a $1 \mathrm{D}$ cross-section of the sun glitter 170 observations.

171 It is important to note that within the surface area where the transfer function 172 becomes zero, $(T=0)$, sun glitter brightness contrasts will change signs. Near the 173 specular point, in the "central" part of the sun glitter, where $T>0$, the rougher surface 
174 patterns will be darker, while far from the specular point, in the "periphery" part, where

$175 T<0$, they will appear brighter. Coordinates of this zone of contrast inversion are found

176 as a solution of $T=0$, which, for the isotropic Gaussian surface slope, simply

177 corresponds to

$$
\left(Z_{x}^{2}+Z_{y}^{2}\right)=s_{0}^{2}
$$

179 where $Z_{x}$ and $Z_{y}$ are given by (2).

180 An example with the transfer function (10) for the isotropic Gaussian PDF

$181\left(\alpha=s_{c}^{2} / s_{u}^{2}=1\right)$ is shown in Fig. 1. The MSS of the sea surface was specified as a periodic

182 oscillation relative to the background value

$$
s^{2}=s_{0}^{2}[1+\varepsilon \cos (2 \pi x / l)]
$$

184 where $l$ is the wavelength of the MSS anomalies, and $\varepsilon$ is the amplitude of the MSS

185 variations. Present calculations are performed for rather "large" MSS variations $(\varepsilon=0.2)$

186 relative to the background value $s_{0}^{2}=3 \cdot 10^{-2}$. Sun and view angles are: $\varphi_{s}=0, \theta_{s}=20^{0}$,

187 and the view angle $\theta_{v}$ varies from $-60^{\circ}$ to $+60^{\circ}$. Sun glitter radiance for the uniform and

188 the disturbed surface (with MSS prescribed by (12)) are shown in Fig. 1a. As it follows

189 from Fig. 1c, "small" ( $\pm 20 \%)$ MSS variations can lead to rather large brightness

190 modulations. The transfer function (10) is shown in Fig. 1b, where the zones of contrasts

191 inversion around $T=0, \theta_{v}=0^{\circ}$ and $\theta_{v}=40^{\circ}$ can be found. Fig. $1 \mathrm{~d}$ demonstrates results

192 of the retrieval of MSS variations. In spite of rather large original modulations of the

193 MSS, prescribed by (13), reconstructed values are quite close to the original both in the

194 central and peripheral parts of the sun glitter. Though some bias of the MSS variations in

195 the peripheral part of the sun glitter can be revealed, the peak-to-trough value of the 
196 retrieved MSS is the same as the original one. Singular behavior of the reconstructed

197 values of the MSS around the zones of the contrast inversion results from the vanishing 198 of the transfer function.

199
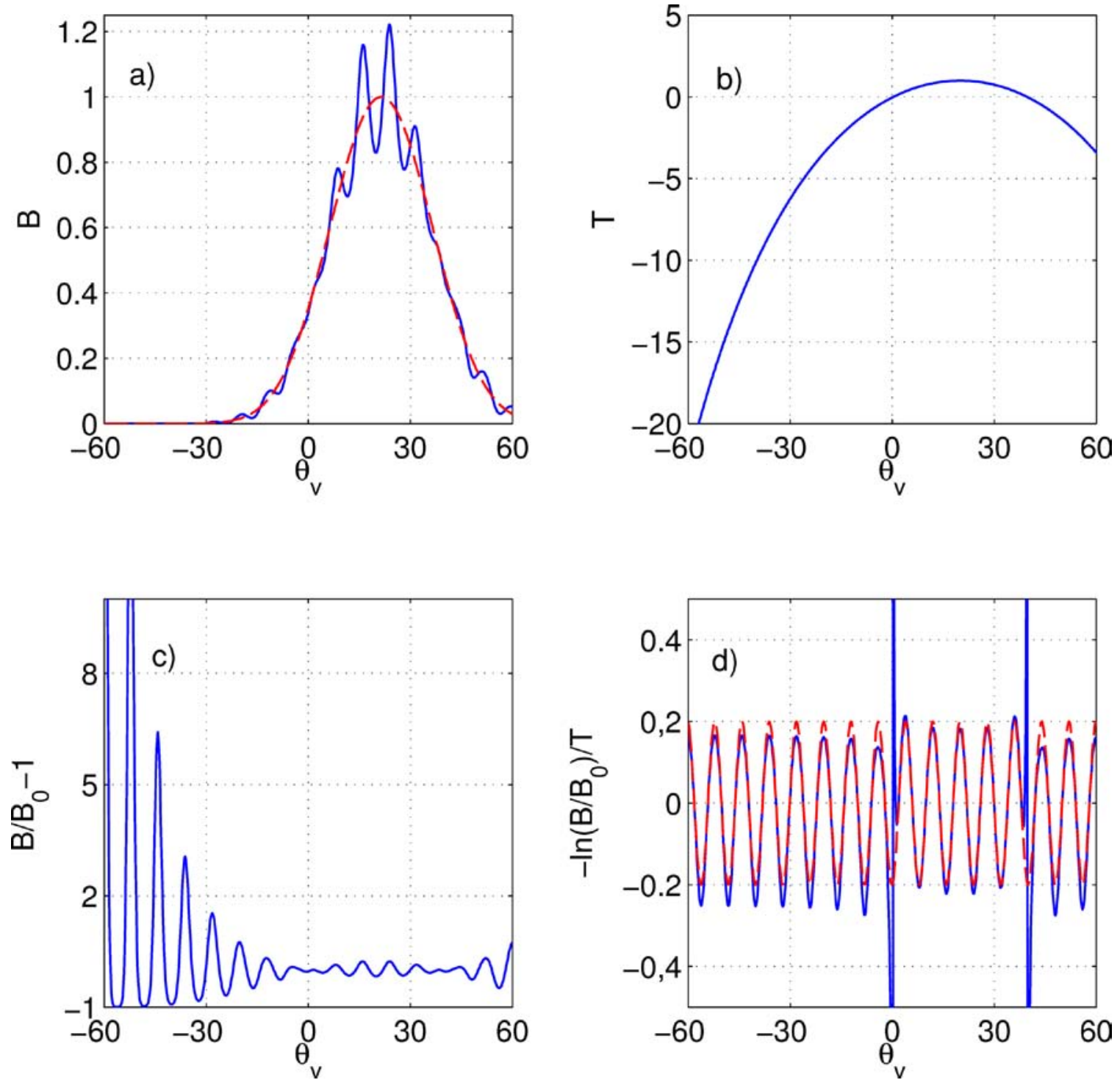

201

Figure 1. a) Sun glitter radiance (in convention units) for the background, $B_{0}$, (dashed

202 line) and disturbed, B, (solid line) surface with MSS prescribed by (12) vs. view angle

$203 \theta_{v}$ (in degrees); b) the Transfer function (10); c) Relative brightness variations, 
$204\left(B-B_{0}\right) / B_{0} ;$ d) solid line,- MSS variations reconstructed from brightness variations

205 shown in plot (c) with use of $T$ shown in plot (b); dashed line, - original MSS variations.

A near-Gaussian PDF is generally expected under moderate wind conditions, but an

208 instantaneous PDF of the sea slopes can be significantly different. Since this "real" PDF

209 predetermines the sun glitter's 2D shape, it is tempting to determine the transfer function

$210 T$ defined by (5b) more directly, without an a priori suggestion for the PDF model.

211 Using (1), the gradients of $p$ in (5b) can be obtained from the "observed" large scale

212 sun glitter brightness gradients

$$
\begin{aligned}
& \frac{\partial \ln p}{\partial \ln \xi}=Z_{x} \frac{\nabla_{x} \ln \left(B_{0} \cos \theta_{v}\right) \cdot \nabla_{y} Z_{y}-\nabla_{y} \ln \left(B_{0} \cos \theta_{v}\right) \nabla_{x} Z_{y}}{\Delta}-\frac{4 Z_{x}^{2}}{1+Z_{x}^{2}+Z_{y}^{2}} \\
& \frac{\partial \ln p}{\partial \ln \eta}=Z_{y} \frac{\nabla_{y} \ln \left(B_{0} \cos \theta_{v}\right) \cdot \nabla_{x} Z_{x}-\nabla_{x} \ln \left(B_{0} \cos \theta_{v}\right) \nabla_{y} Z_{x}}{\Delta}-\frac{4 Z_{y}^{2}}{1+Z_{x}^{2}+Z_{y}^{2}}
\end{aligned}
$$

214 where $\left(\nabla_{x}, \nabla_{y}\right)$ are the gradients in the $(x, y)$ directions, and $\Delta$ is the discriminant:

$215 \Delta=\nabla_{x} Z_{x} \cdot \nabla_{y} Z_{y}-\nabla_{y} Z_{x} \cdot \nabla_{x} Z_{y}$. This approach is thus self-consistent. The large-scale

216 2D shape of the sun glitter brightness, $B_{0}(x, y)$, defines the transfer function $T(x, y)$ (via

217 eqs. (5b) with (13)) which is then used for conversion of the brightness variation

$218 \tilde{B}=B-B_{0}$ into the MSS contrasts following eq. (5a). Note, that this self-consistent

219 approach for the MSS anomalies retrieval is similar to the method of reconstruction of $2202 \mathrm{D}$ spectrum of the dominant surface wave slopes from photographs of the sun glitter 221 suggested by Bolshakov et al. (1990a; 1990b). 
Due to the scanning mirror construction, the MODIS image represents a composition

224 of stripes. Each one is formed by 40 detectors with the along track field of view of about

$2250.8 \mathrm{deg}$ and cross track field of view of about $110 \mathrm{deg}$. This instrument viewing geometry

226 yields a cross-track length of $2330 \mathrm{~km}$ with a width of about $10 \mathrm{~km}$ at nadir. Each stripe

227 provides a $2 \mathrm{D}$ field of the surface brightness. Having the $2 \mathrm{D}$ field of the surface

228 brightness available, the above method can be applied, and operators $?_{x}$ and $\nabla_{y}$ in (13)

229 become along- and cross-stripe gradients, respectively.

230 In case of MERIS imagery, only cross-track gradients of the surface brightness are

231 available. Therefore, we are inevitably forced to use an a priori PDF model. The sun

232 glitter model (1) with (8) can be rewritten as

$$
Y=-X / s_{0}^{2}+C
$$

234 where $Y=\ln \left(B_{0} \cos \theta_{v} \cos ^{4} \beta\right), \quad X=a_{2} Z_{x}^{2}-2 \mathrm{a}_{12} Z_{x} Z_{y}+a_{1} Z_{y}^{2}$, and $C$ is a "constant"

235 taking into account other model parameters $\left(E_{0}, \rho, \alpha\right.$, and $\left.s_{0}^{2}\right)$. A mean value of the

$236 \operatorname{MSS}\left(s_{0}^{2}\right)_{k}$ for each line (of index $\mathrm{k}$ ) of the image can then be estimated using the 237 observed brightness, as a solution of (14). The mean square root method then yields

$$
\left(1 / s_{0}^{2}\right)_{k}=-\frac{\sum_{j}\left(Y_{j k}-\bar{Y}_{k}\right)\left(X_{j k}-\bar{X}_{k}\right)}{\sum_{j}\left(X_{j k}-X_{k}\right)^{2}},
$$

239 where the overbars denote the mean (averaged over a cross-track k-line) values. Once $s_{0}^{2}$

240 is estimated, the contrasts are evaluated from (5a) with the transfer function defined by $241(10)$ 


\section{Imagery of the surface slicks}

\subsection{Natural oil slicks}

We first consider the MODIS images of the Gulf of Mexico possessing distinct sun

245 glitter brightness features related to the mineral oil spills. Investigating these images, $\mathrm{Hu}$ 246 et al. (2009) found that the sun glitter contrasts of the surface slicks appear to be either

247 dark or bright. The sign of the slick contrasts was reported to depend on the angle

248 between the viewing direction and the direction of the mirror reflection, $\theta_{m}$. Slick

249 contrasts in an examined case changed sign at $\theta_{m} \approx 12^{\circ}$, being positive at smaller angles

250 and negative at larger angles. Hu et al. (2009) questioned whether this observation could

251 be generalized, and recommended further research.

252 A fragment of the original MODIS image (MODIS/Terra, 2 June 2005, 16:55 GMT) 253 analyzed by $\mathrm{Hu}$ et al. (2009) is shown in Fig. 2 (upper left). This image contains

254 numerous curved brightness features. The brightness contrasts $\widetilde{B} / B_{0}$ presented in Fig. 2 255 (upper right) have different sign on the opposite side of $92^{\circ} \mathrm{W}$. The origin of this zone of 256 contrast inversion follows from the definition of the transfer function (5b). As mentioned 257 above, the line dividing the sun glitter area in two parts, where the MSS variations lead to 258 negative or positive contrasts, follows from solution of the equation: $T(x \cdot y)=0$.

259 Fig. 2 (lower left) shows the transfer function calculated using (5b) and (13) for the

260 smoothed sun glitter brightness field. The contrasts $\tilde{s}^{2} / s_{0}^{2}$ retrieved from the brightness

261 field are shown in Fig. 2 (lower right). In the vicinity of the zone of the contrast 262 inversion, where the transfer function $T \rightarrow 0$, it appears as a $z$ one of "singular" large 263 values of contrasts (which have no physical meaning). 
As derived, contrasts likely associated with oil slicks are now systematically

265 negative. One can also notice the other type of the MSS features (both positive and 266 negative) which presumably caused by the wind field variability on the inner scale. The

267 MSS contrasts associated with the oil slicks are about $s^{2} / s_{0}^{2} \approx 0.3-0.4$, that is equivalent 268 to a reduction of the MSS in the oil slick by factor of 1.5. This estimate is lower than the 269 MSS reduction by factor $2-2.5$ reported by Cox and Munk (1954) for the surface slicks 270 produced by a mixture of fish oil, crankcase oil and diesel. Note that the elasticity of such 271 a surface film is dominated by the elasticity of the fish oil which is about $30 \mathrm{mN} / \mathrm{m}$, 272 presumably larger than the elasticity of the mineral oil film, which is poorly known (one 273 of the suggested estimates is $E=4 \mathrm{mN} / \mathrm{m}$, personal communication by S. Ermakov). Since 274 the elasticity of the surface films determines the suppression of short wind waves, a 275 smaller elasticity of the surface film should lead to a smaller contrast in slicks (see sec. 276 3.3. and Fig. 11 below).

277 Fig. 3 (left) shows an enlarged fragment of Fig. 2 (lower left) containing "individual" 278 oil slicks, and Fig. 3 (right) presents the relationship between MSS contrasts and wind 279 speed of the twelve selected oil slicks. The estimates of wind speed were obtained from $280 s_{0}^{2}$ converted to the wind speed following the empirical relationship by Cox and Munk 281 (1954). Fig. 3 further shows the MSS contrast of the biogenic slicks reported by Cox and 282 Munk (1954, page 847); it is defined as ratio of the MSS regression lines for clean and 283 slicks areas. As it follows from Fig. 3 (right), at low wind speeds, the observed MSS 284 contrasts of mineral oil slicks are consistent with the contrasts reported by Cox and Munk 285 (1954) for fish oil slicks, but, at moderate wind speeds $(>4 \mathrm{~m} / \mathrm{s})$, the contrasts from 286 mineral oil slicks are systematically lower than those from fish oil. 

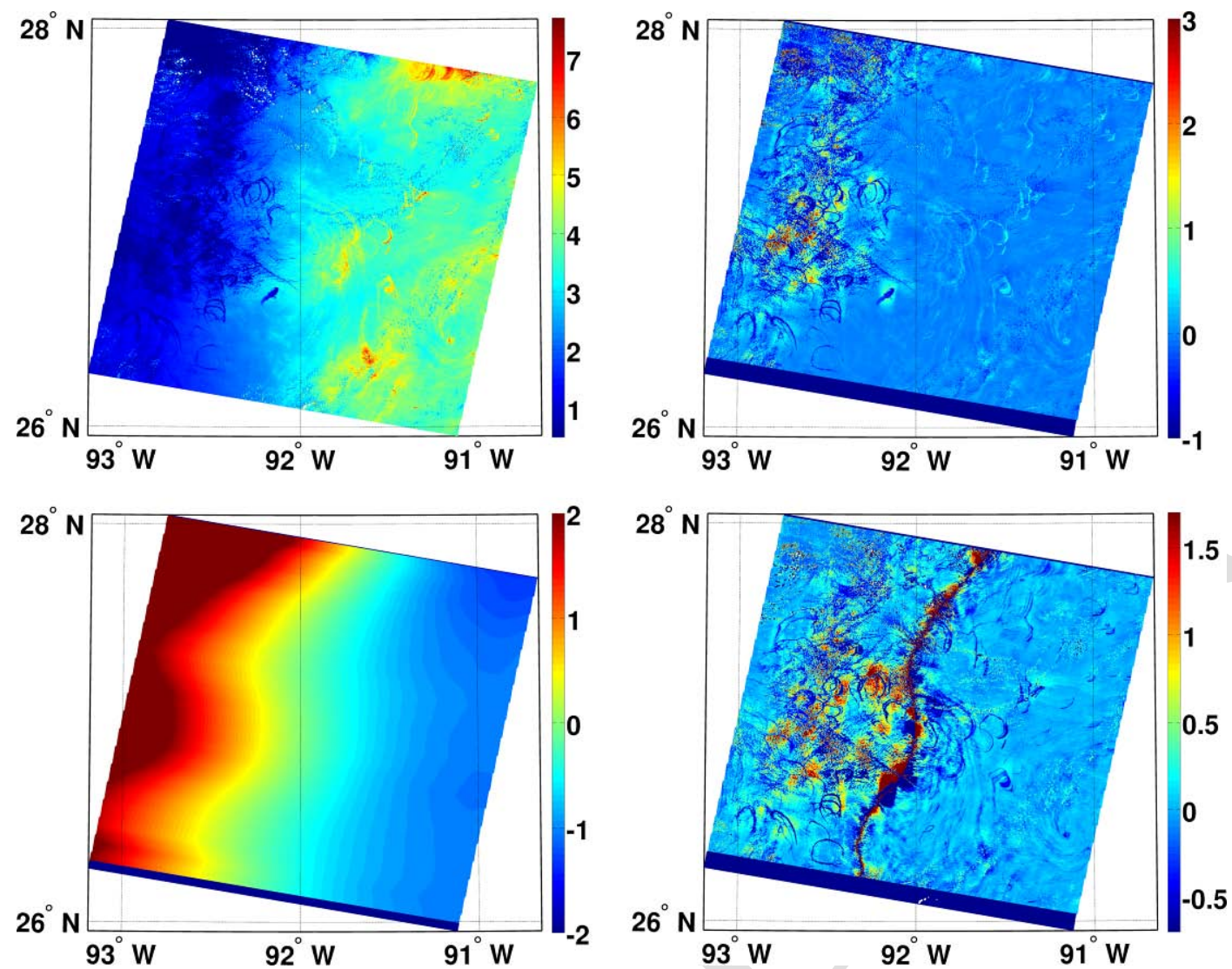

289 Figure 2. (upper left) Fragment of the MODIS/Terra image (June, 2, 2005, 16:55 GMT)

290 in the $645 \mathrm{~nm}$ (red) channel of the Gulf of Mexico with sun glitter signatures of mineral

291 oil spills. (upper right) The brightness contrasts $\widetilde{B} / B$. (lower left) The transfer function.

292 (lower right) Retrieved MSS contrast.

293
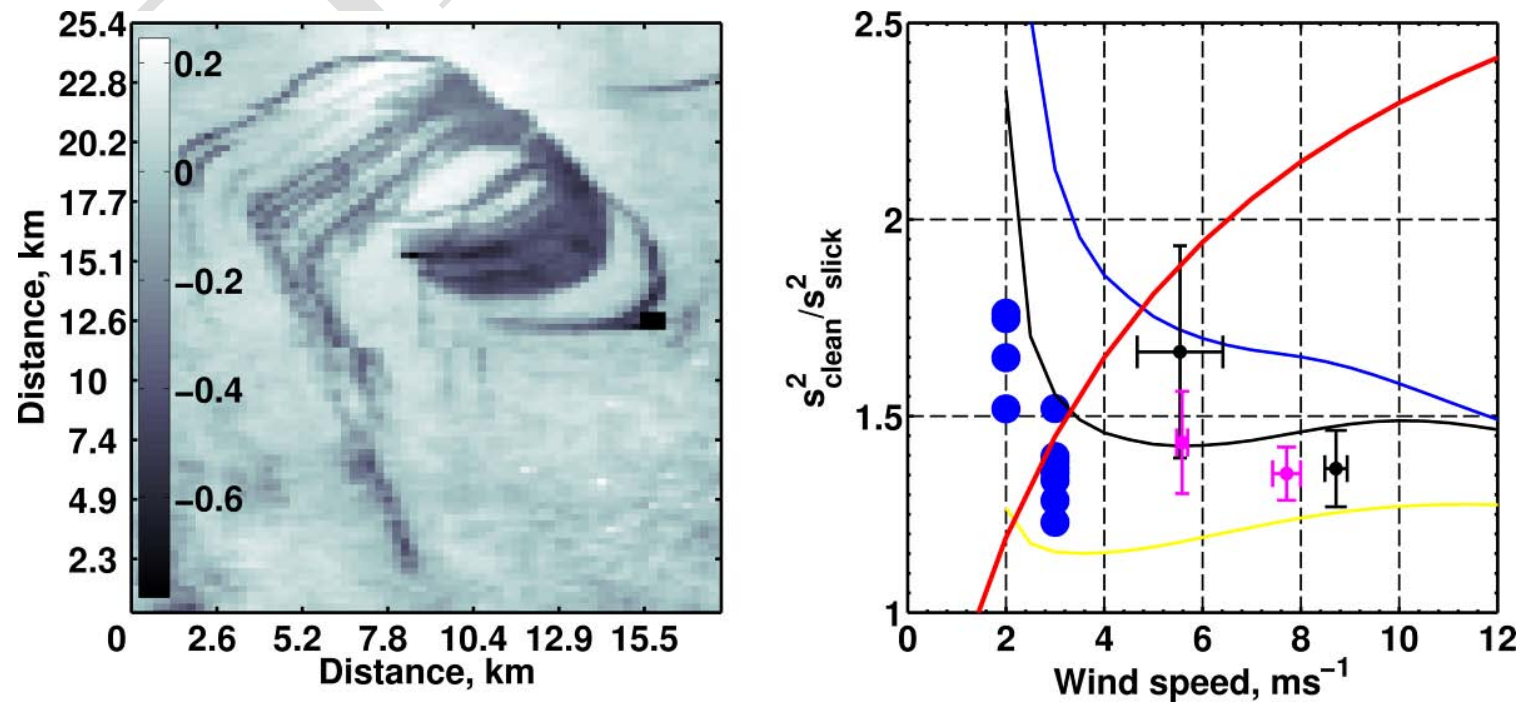
295 Figure 3. (left) Enlarged fragment of the MSS contrasts taken from Fig. 2 containing

296 "individual" oil slicks. (right) MSS contrasts of the oil slicks derived from the MODIS

297 image in Fig. 2 (blue circles around wind speed 2-3 m/s), from the MERIS images shown

298 in Fig. 7 and Fig. 9 (pinky circles with error bars), and from the MODIS images (black

299 circles with error bars). The red line represents MSS contrasts of the fish oil slicks as

300 reported by Cox and Munk (1954). Yellow, black and bl ue lines are the model

301 simulations of the MSS contrasts of the surface slicks caused by thin surface film with

302 elasticity 5,15 and $30 \mathrm{mN} / \mathrm{m}$ respectively.

\subsection{Catastrophic oil spills}

305 The Deepwater Horizon oil spill on A pril 20,2010 i s chosen for further 306 demonstration. The MODIS (MODIS/Terra, May, 24, 2010, 16: 45 GMT) and MERIS 307 (MERIS/Envisat, May 24, 2010, 16:17 GMT) images from the red channels (645nm and $308681 \mathrm{~nm}$ correspondingly) are shown in Fig. 4. Note the oil spill is not entirely covered by 309 the MODIS/Terra image. The image shown in Fig. 4 (lower) is a composition of two 310 MODIS/Terra images acquired on 16:45 and 16:50 GMT. The time difference between

311 these MERIS and MODIS acquisitions is about half an hour, therefore "geometry" of the 312 oil spill on the ocean surface should not been changed during this period. As evidenced, 313 due to different viewing and sun angles, the spill signatures on the MODIS and MERIS 314 images are quite different. 

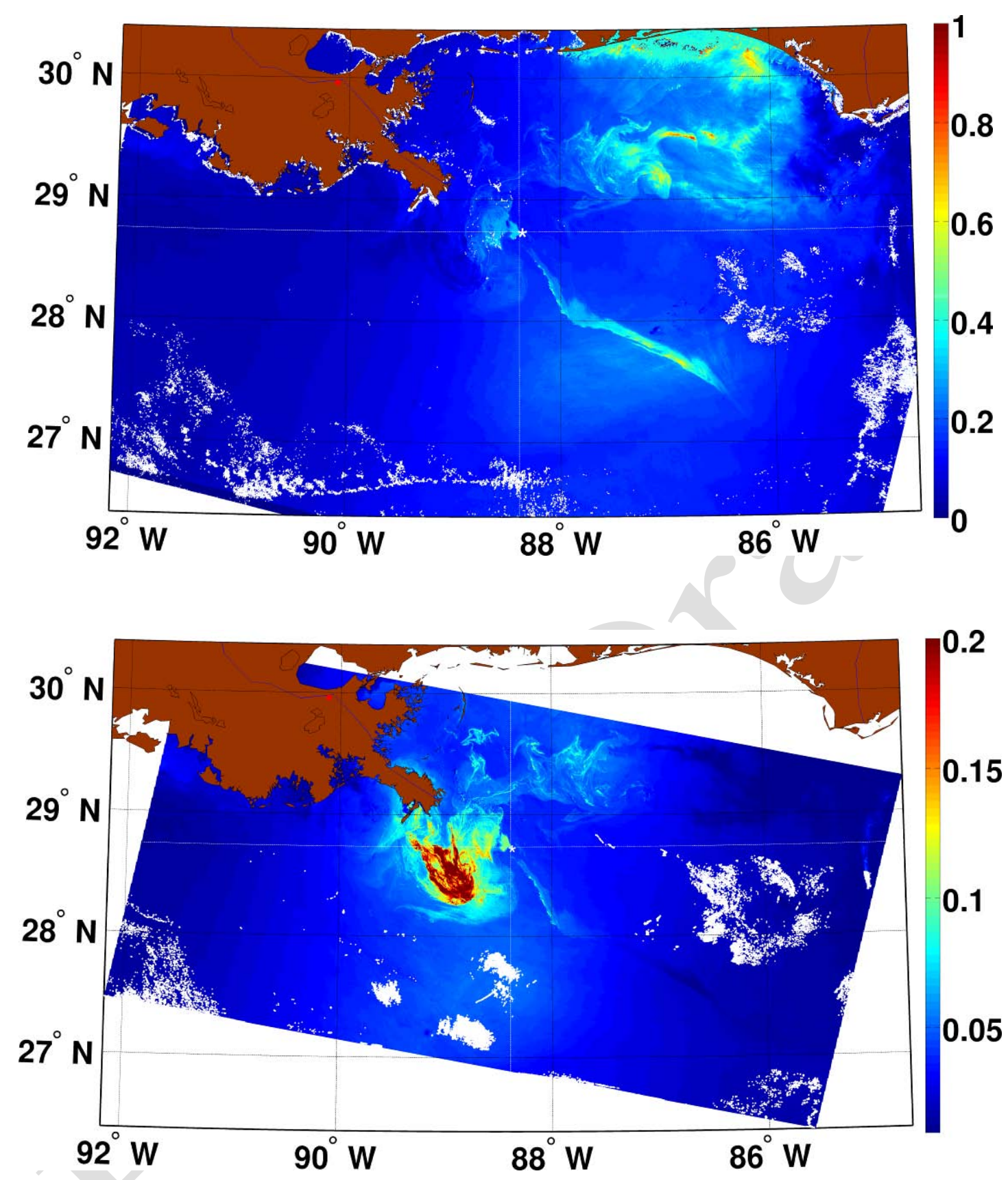

Figure 4. (upper) Fragments of the original MERIS/Envisat image in red channel (681nm) acquired on May 24, 2010, 16:17 GMT. (lower) Composition of two MODIS/Terra images in red channel (645nm) acquired on May 24, 20 10, 16:45 GMT and 16:50 GMT correspondingly. Color bars indicate radiance of the images in conventional units. Cloud mask is shown with white and land mask with brown 
colors. Coordinates of the Deepwater Horizon Platform are $28.73^{\circ} \mathrm{N}, 88.38^{\circ} \mathrm{W}$

317 The images were processed using the methodology described in Sec.2. Fields of the

318 mean sun glitter brightness $B_{0}$ (averaging scale is $30 \times 30 \mathrm{~km}^{2}$ ) for MERIS and MODIS

319 data are shown in Fig. 5 (upper left) and 5 (upper right). The transfer function for the

320 MODIS data is directly calculated from the mean brightness field (following eqs. (5b)

321 with (13)), and is shown in Fig. 5 (lower right). Notice that an inclined linear

322 discontinuity well visible in this figure results from the patching of two MODIS/Terra

323 images. To assess the transfer function for the MERIS data, the wind direction was

324 prescribed from NCEP data, and the mean MSS was then calculated following eq.(10).

325 The transfer function for MERIS data is show in Fig. 5 (lower left).
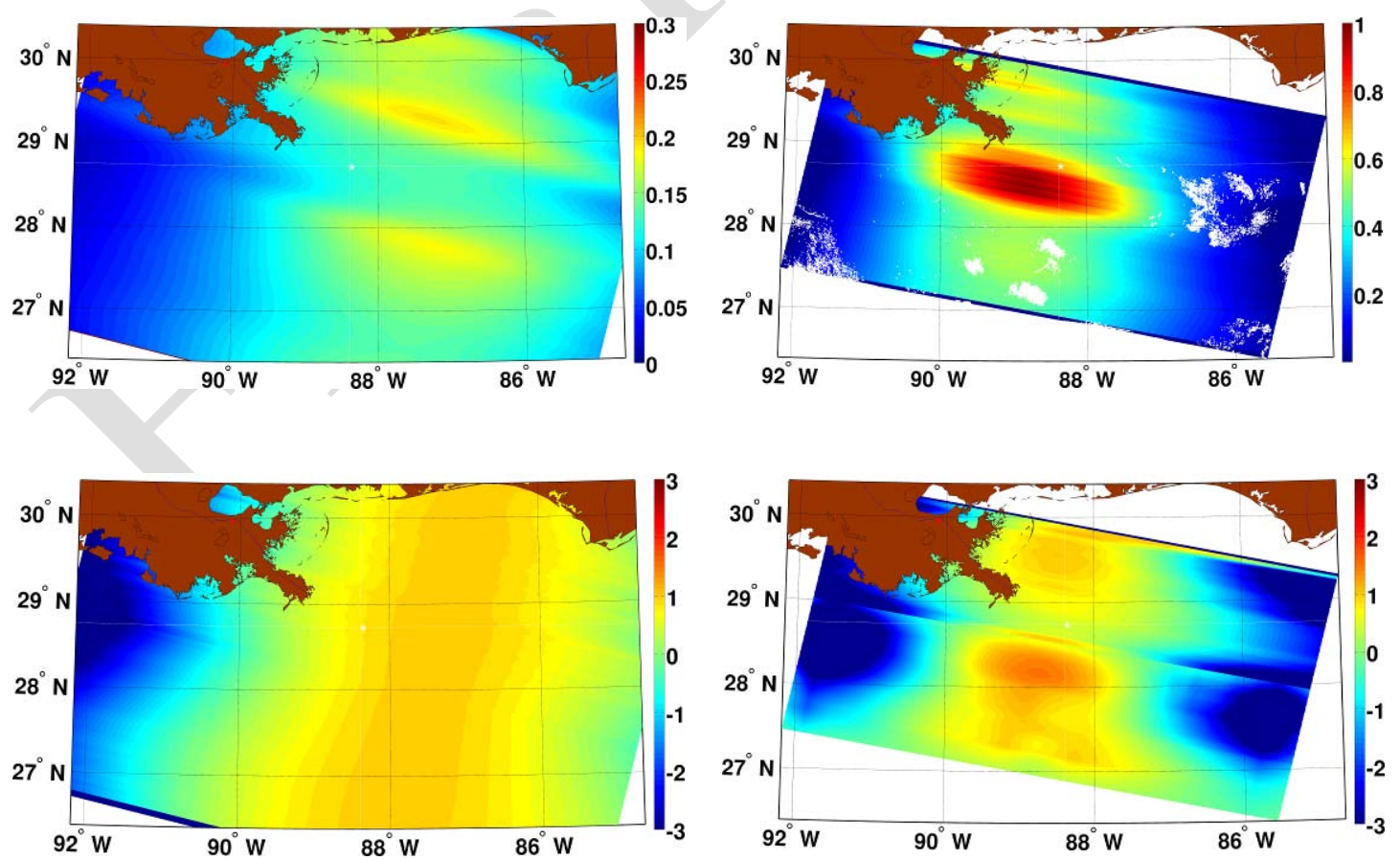
327 Figure 5. (upper left), (upper right) The averaged brightness $B_{0}$ of the MERIS and 328 MODIS images correspondingly. (lower left), (lower right) The transfer function $T$ 329 defined by eq. (10) for MERIS and by eq. (5b) with (13) for MODIS. An inclined linear 330 discontinuity in the field of $T$ in plot (lower right) around $28.5^{\circ} \mathrm{N}$ results from the 331 patching of two MODIS/Terra images acquired on 16:45 and 16:50 GMT.

333 The sun glitter brightness contrasts $\tilde{B} / B_{0}$ for the MERIS and MODIS data are 334 shown in Fig. 6. The brightness contrasts fields are in close agreement. Some apparent 335 differences are still evident. The contrast feature which is the "oil jet" around $87^{\circ} \mathrm{W}$ is 336 viewed as a bright jet in the MERIS image (Fig. 6 (upper)), but in the MODIS field 337 (Fig. 6 (lower)), the jet varies from bright to dark. Referring to Fig. 5 (upper right), one 338 can see that the transfer function $T$ changes sign in this area, which corresponds to the 339 zone of the inversion of brightness contrasts. The oil jet crosses the area of the contrast 340 inversion zone, and thus its sun glitter brightness signature in the MODIS images 341 changes sign. 

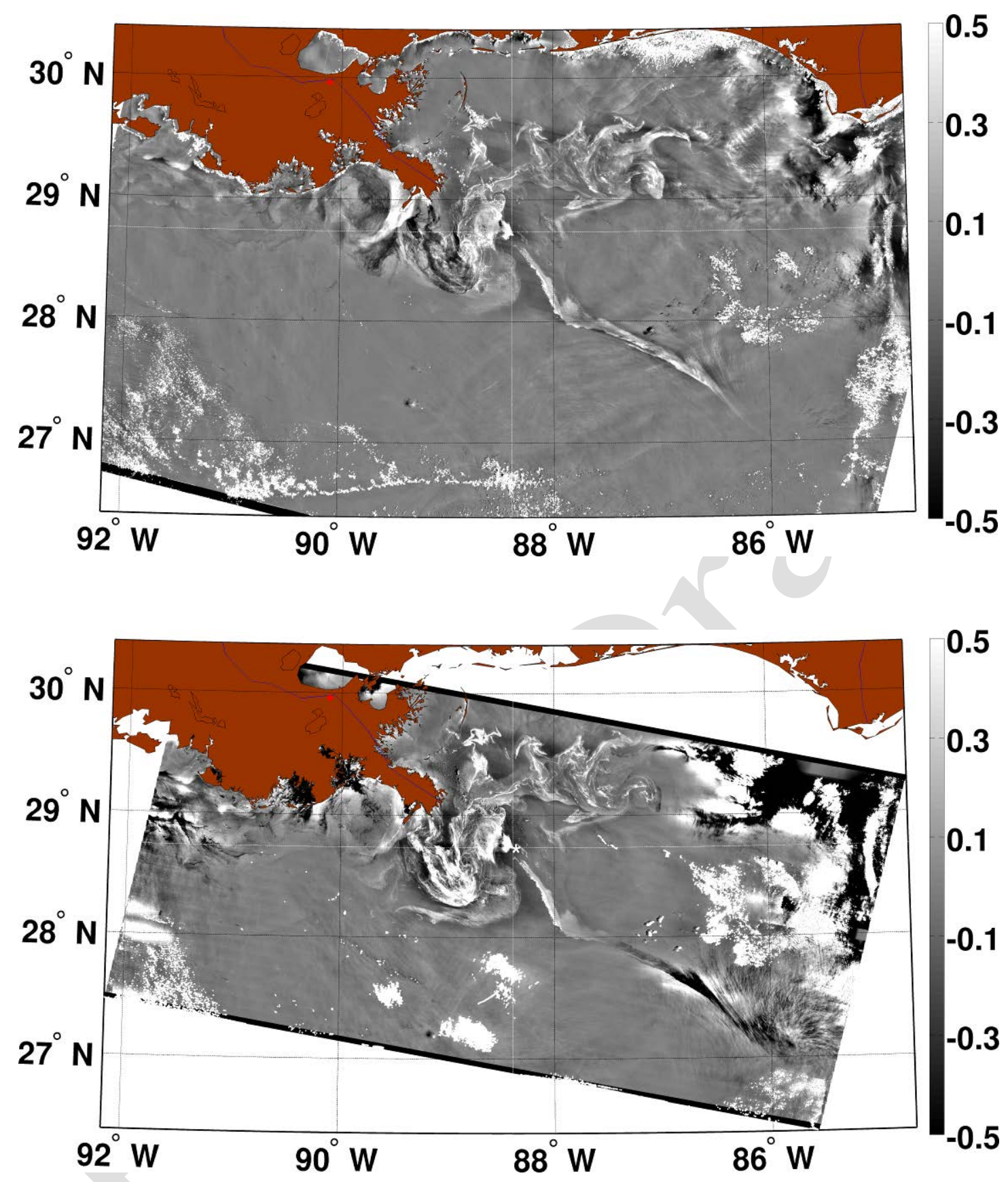

Figure 6. Sun glitter brightness contrasts, $\widetilde{B} / B_{0}$, in the MERIS (upper) and MODIS (lower) images 
343 Fig. 7 shows the MSS contrasts $\tilde{s}^{2} / s_{0}^{2}$, derived from the MERIS and MODIS sun

344 glitter brightness contrasts (Fig. 6) with use of the transfer functions presented in Fig. 5.

345 It was found that the MSS anomalies derived from the MERIS and MODIS images are in

346 good agreement, with magnitudes of the MSS contrasts of the same order. The MSS

347 anomalies derived from two independent images with the use of two different methods

348 show very similar results. This proves the robustness of the proposed methodology. The

349 averaged MSS contrasts in the identified jet derived from the MODIS and MERIS

350 images are shown in Fig. 3 (right) above.

$351 \quad$ Few remarkable differences are also found. First, linear features (indicated by red 352 arrows) with singular values trace the zones of the inversion of contrasts. Other 353 differences, confined within the yellow contour, in the vicinity of the mouth of the 354 Mississippi River, present both negative and positive values. Moreover, these 355 positive/negative values in both images do not overlap. Considering that an oil film 356 suppresses the short waves and the MSS, the "bright" MSS features in Fig. 7 must be 357 considered as artifacts caused by other factors. We can anticipate that the oil film's 358 thickness in this area may be thick relative to the wavelength of red light $(640 \mathrm{~nm}$ $359680 \mathrm{~nm})$, i.e. with thickness of order $5-50 \mu \mathrm{m}$ or more. In this case, the radiance of the 360 surface is dominated by the optical properties of the oil itself. The suggested algorithm 361 does not take this effect into account, and the reconstructed contrasts are not valid in 362 either magnitude or sign change. 

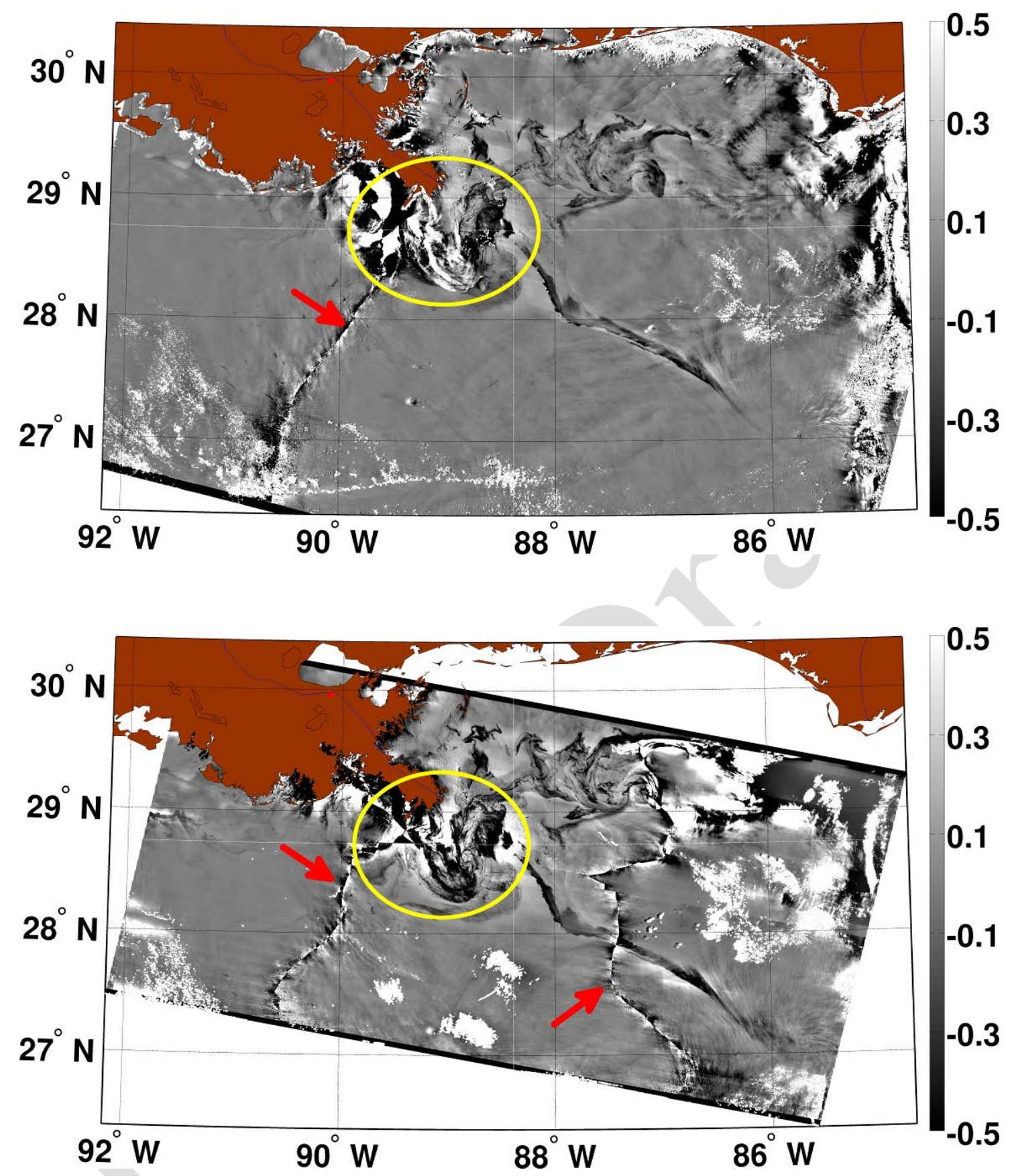

364 Figure 7. The MSS anomalies $\widetilde{s^{2}} / s^{2}$ derived from the MERIS (upper plot) and MODIS

365 (lower plot) images. Red arrows indicate zones of the inversion of contrasts where 366 reconstructed MSS have singular values (without physical meaning). MSS anomalies

367 confined to the yellow contours are presumably not true, because the oil film thickness in 368 this area is too large relative to the red light wavelength. Since the considered method 
does not take this effect into account, bright/dark features inside the yellow contours

should be considered artifacts.

To further illustrate the effect of oil thickness, we consider a case where synchronous

373 MERIS and ENVISAT Synthetic Aperture Radar (ASAR) images are available. Fig. 8

374 shows ASAR (in terms of wind speed) and MERIS (red channel) images of the Gulf of

375 Mexico region on April, 26, 2010. T he oil spill is visible in both images. Zoomed in

376 Fig. 9, the fields of the Normalized Radar Cross Section (NRCS) (in linear units) and

377 MSS contrasts are presented. The MSS contrasts were derived following the method 378 discussed above.
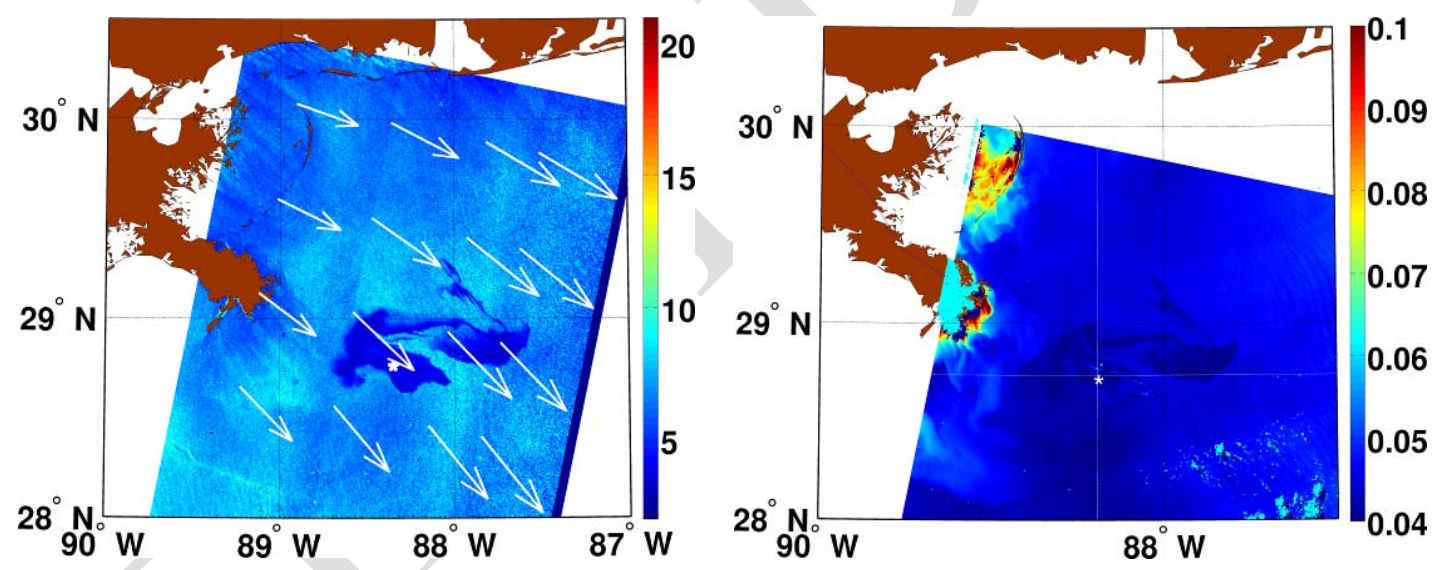

380 Figure 8. ASAR (15:58 GMT), left, and MERIS (15:56.GMT), right, red channel images

381 of the oil spill in the Mexican Gulf on April, 26, 2010. A SAR is represented in terms of 382 wind speed derived from the NRCS with use of CMOD-4 function. White arrows in SAR 383 wind indicate NCEP wind direction.

As derived, the spill signature clearly exhibits a very similar geometrical shape, but 386 obtained contrasts can be very different. Divided into two parts (within and outside the 
387 yellow contour), the 'outside' NRCS and MSS contrasts agree well. A scatter plot of the

388 MSS and NRCS contrasts is shown in Fig. 10. Correlation is obvious, with MSS contrast 389 magnitudes slightly lower than the NRCS ones by a factor of 0.6 , $\mathrm{i}$.e. $390 \tilde{s}^{2} / s^{2} \approx 0.6 \cdot \tilde{\sigma}_{0} / \sigma_{0}$.

391 The MSS contrast averaged over this part of the slick is also reported in Fig. 3 (right).

392 Note that the same slick was observed by MODIS a half hour later (not shown here).

393 Processing of this image yields MSS anomalies very similar to those shown in Fig. 9, and 394 the averaged value also reported in Fig. 3 (right).

395 The averaged NRCS contrast of the oil slick outside the yellow contour in Fig. 9 396 (upper) is given in Fig. 10 (right). Two other estimates of the NRCS contrasts in Fig. 10 397 (right) at lower wind speeds are obtained from an ASAR image on May 25, 2010, 15:47

398 GMT (not shown here), i.e. the day after the MERIS and MODIS images discussed in 399 Fig. 4 were acquired. In both cases wind speed is derived from original ASAR images 400 with use of CMOD-4 function (see Fig. 8 left as example). These estimates of the NRCS 401 contrasts clearly indicate their strong dependence on w ind speed. Comparing NRCS 402 contrasts in Fig. 10 (right) with MSS contrasts in Fig. 3 (right) reveals that, under 403 relatively low to moderate wind speed conditions, oil slicks are more visible in SAR 404 images than in optical ones. 

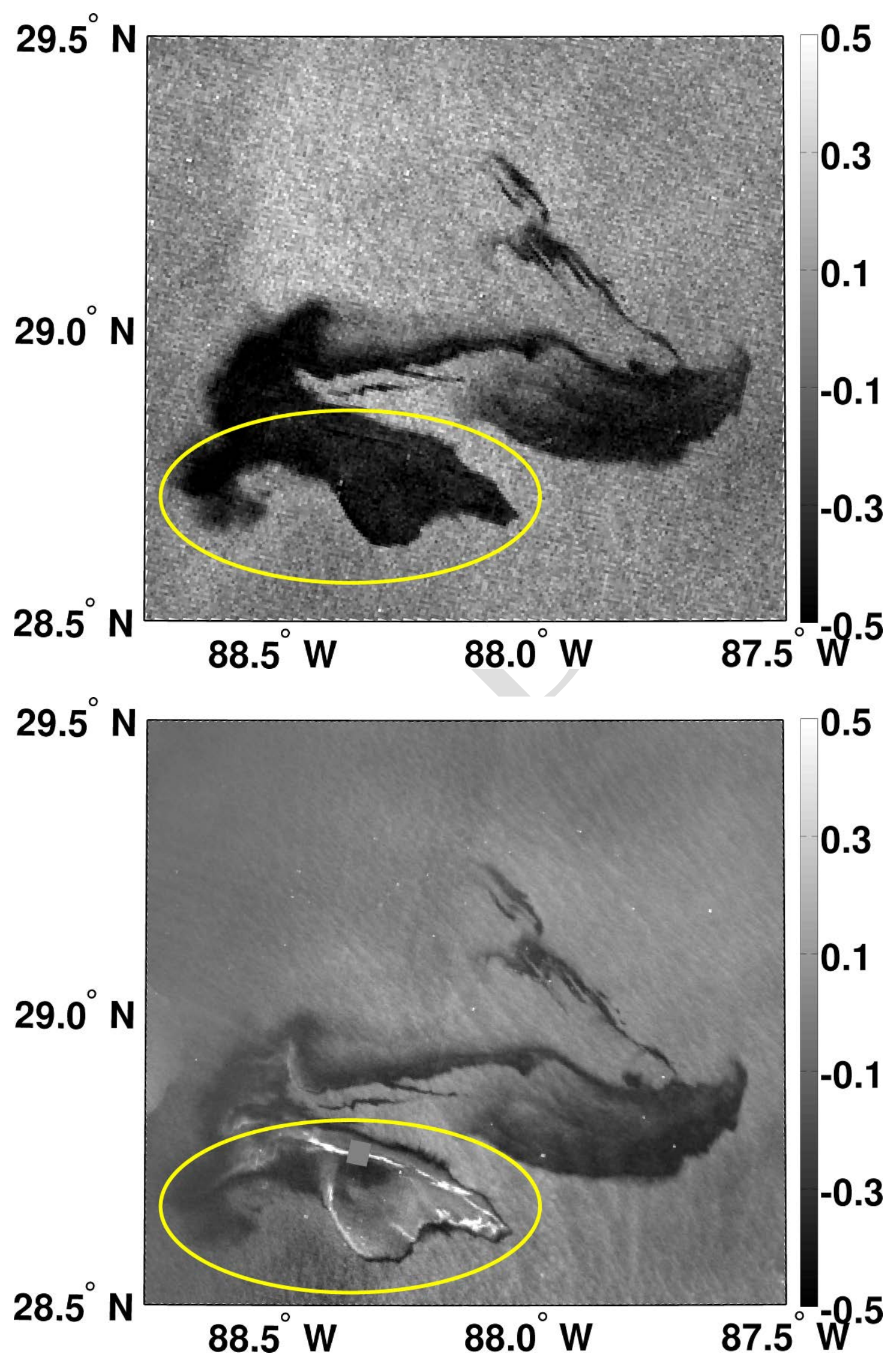

406 Figure 9. Enlarged fragments of the ASAR (upper) and MERIS (lower) images shown in 
407 Figure 8 containing the oil spill, and represented in terms of the NRCS (linear units) and

408 MSS contrasts. The yellow contour confines the slick area where the oil film thickness is

409 presumably large relative to the red light wavelength.

410

411 Revisiting Fig. 9, the area inside the yellow contour appears anomalous. While the

412 NRCS is still suppressed in this area, the MSS contrasts exhibit strong variability, and

413 some of the patches are bright. SAR data undoubtedly indicate that short wind waves are

414 strongly damped. Accordingly, the bright patches of the MSS contrast do not relate to the

415 surface roughness peculiarities, but are likely indicating the influence of the optical

416 properties of the oil film. The oil film thickness in this area may be thought to be

417 significantly larger than the red light wavelength. We may then assume that the bright

418 linear features which are well visible inside the yellow contour more directly correspond

419 to the oil color.

420
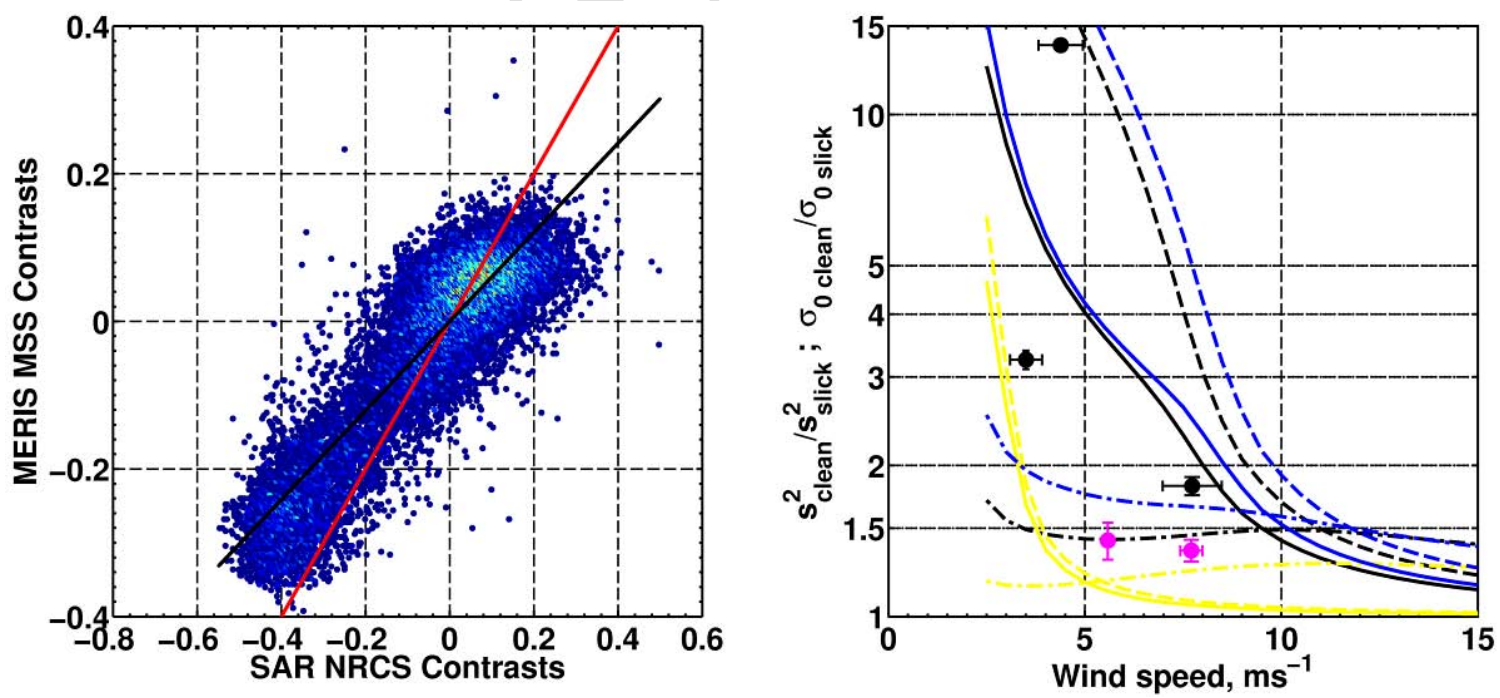

422 Figure 10. Left: the MSS contrasts vs. the NRCS contrasts of the oil slick in the area

423 outside the yellow contour in Fig. 9. The red line indicates a one-to-one relation, and the

424 black line is the linear fit of the data: $\tilde{s}^{2} / s^{2} \approx 0.6 \cdot \tilde{\sigma}_{0} / \sigma_{0}$. Right: black dot with the error 
425 bars at wind speed $7.5 \mathrm{~m} / \mathrm{s}$ is the averaged NRCS contrasts of the oil slicks (outside the

426 yellow contour) shown in Fig. 9. Two other black dots are the NRCS contrasts of the

427 same oil spill derived from ASAR image acquired on May 25, 2010, 15 :47 GMT (not

428 shown here). Pinky dots are the averaged MSS contrasts derived from MERIS image and

429 shown in Fig. 7 (upper), for oil jet, and in Fig. 9 (lower) for the area outside the yellow

430 contour. Dash-dotted lines are RIM simulation of the MSS contrasts of oil slick with

$431 E=5,15,30 \mathrm{mN} / \mathrm{m}$ (yellow, black and blue color lines correspondingly). RIM simulation

432 of the NRCS contrasts of oil slick contrasts within the frame of a "pure" Bragg

433 scattering model and full NRCS model accounting for the effect of wave breaking on

434 radar backscatter are shown by dashed and solid lines, respectively. Color style of the

435 NRCS contrasts lines is the same as that of the MSS.

\subsection{MSS and NRCS contrasts of oil slicks}

438 As obtained and shown in Fig. 3 (right), the MSS contrasts are believed to correspond 439 to oil film that is thin relative to the red light wavelength. The thickness is certainly much 440 smaller than the capillary wave wavelength, and the damping mechanism of surface 441 waves by this thin oil film can be described within the frame of classical Marangoni 442 theory (Levich, 1962). In this case, the modulus of elasticity is the only and yet poorly

443 known parameter characterizing damping properties of thin, mineral oil film.

444 The oil film on w ind waves acts through the modification of the wave damping 445 coefficient. Relation for the wave damping coefficient in the presence of surface thin film 446 is given in Levich (1962), and also reproduced in sec. 4.2 in Kudryavtsev et al. (2005).

447 Viscous dissipation plays a key role in the energy balance of capillary-gravity waves, 448 leading surface films to increase the energy dissipation and to affect both the short wave 
449 spectrum and the MSS. Following the radar imaging model (RIM) suggested in

450 Kudryavtsev et al. (2005) and Johannessen et al. (2005), the energy balance in the

451 equilibrium range of gravity and capillary-gravity waves is written as

$$
\beta_{v}(\boldsymbol{k}) B(\boldsymbol{k})-B(\boldsymbol{k})[B(\boldsymbol{k}) / \alpha]^{n}+I_{w b}(\boldsymbol{k})=0
$$

453 where $B(\boldsymbol{k})$ is the saturation spectrum of wind waves, $\alpha$ and $n$ are the model

454 parameters, $I_{w b}$ rate of the energy input to short waves due to breaking of longer wind

455 waves (including generation of parasitic capillaries), $\beta_{v}$ is an effective growth rate

$$
\beta_{v}=c_{\beta}\left(u_{*} / c\right)^{2} \cos \varphi|\cos \varphi|-4 v k^{2} / \omega
$$

457 representing the difference between wind energy input (first term on the 1.h.s.) and 458 viscous dissipation (second term on $\mathrm{t}$ he 1.h.s.), $\varphi$ is angle between wind and 459 wavenumber vector directions, $c, \omega$ and $\boldsymbol{k}$ are the phase velocity, frequency and 460 wavenumber vector correspondingly, $c_{\beta}$ is wind wave growth rate "constant", $u_{*}$ is air 461 friction velocity, $v$ is an effective viscosity coefficient which takes into account effect of 462 the surface film (for the clean surface $v$ corresponds to the molecular viscosity 463 coefficient of the water, $\left.v_{0}\right)$. Equation (17) states that the short wind wave spectrum 464 results from the balance of different sources and sinks of the energy, represented in (17) 465 by the wind energy input and viscous dissipation (first term), non-linear energy losses 466 including wave breaking (second term) and generation of the bound (parasitic capillaries) 467 and free short waves by breaking of longer wind waves (third term). Shape of the wave 468 spectrum results from solution of equation (17) (see Kudryavtsev et al., 2005 for more 469 details).

470 The effective viscosity coefficient of the water surface covered by thin surface film 
471 with the elasticity $E=5,15$, and $30 \mathrm{mN} / \mathrm{m}$ normalized by the water viscosity coefficient

472 is shown in Fig. 11a. With increasing film elasticity the magnitude of the effective

473 viscosity coefficient increases and its peak shifts toward the longer waves. Apparently,

474 an enhancement of the viscous dissipation disturbs the energy balance (17) and leads to

475 suppression of spectral energy in the high frequency range. The omnidirectional

476 (integrated over all directions) spectra in the presence of surface films are shown in

477 Fig. 11b. A remarkable feature of these spectra is appearance of the spectral cutoff that

478 follows the lower-frequency shift of the effective viscosity with increasing elasticity.

479 This spectral cutoff is apparently related to the zero crossing of the effective growth rate

480 (18), when viscous dissipation (depending on $E$ ) exceeds wind energy input.

481 Wavenumber of the spectral cutoff $k_{c u t}$ can be evaluated from the solution of the

482 equation $\beta_{v}\left(k_{c u t}\right)=0$ in, e.g. wind direction. The MSS of the sea surface is expressed via

483 the omnidirectional saturation spectrum as

$$
s^{2}=\int_{k} B(k) d \ln k
$$

485 As follows from this equation, the MSS should be sensitive to the wavenumber of the 486 spectral cutoff, and thus can be used for assessment of the film elasticity from the 487 contrast of the MSS over a slick. Note also the local high-frequency spectral peaks in the 488 saturation spectra for the films with $E=5$ and $15 \mathrm{mN} / \mathrm{m}$. These peaks result from 489 generation of parasitic capillaries by breaking of short gravity waves. In case of $490 E=30 \mathrm{mN} / \mathrm{m}$, these short gravity waves are significantly damped by the film that prevents 491 generation of the parasitic capillaries. 

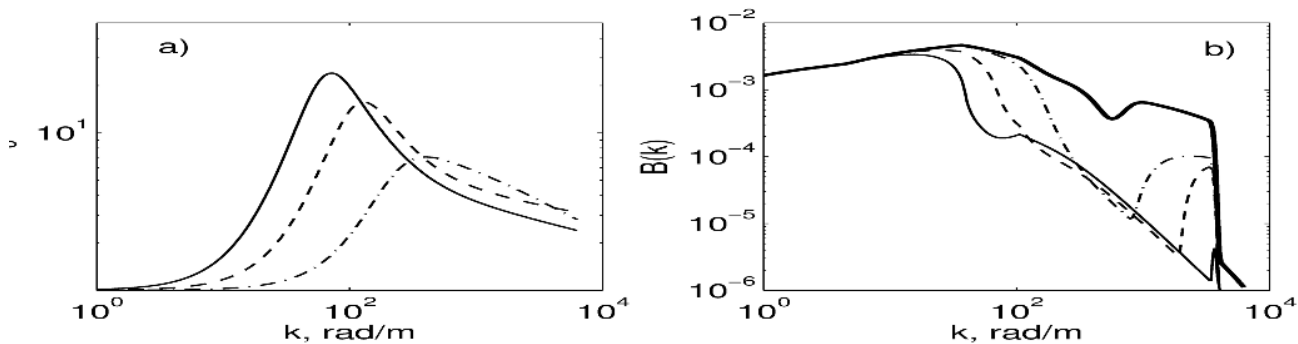

494 Figure 11. a) Wave dumping coefficient, $v$, scaled by the water viscosity, $v_{0}$, for the

495 surface films of different elasticity, $E,: E=5 \mathrm{mN} / \mathrm{m}$ (dash-dotted), $E=15 \mathrm{mN} / \mathrm{m}$ (dashed),

496 and $E=30 \mathrm{mN} / \mathrm{m}$ (solid). b) Omnidirectional saturation spectra of wind waves for the

497 clean surface (thick solid, reference spectrum), and the surface covered by film of

498 elasticity by covered by thin film with $E=5 \mathrm{mN} / \mathrm{m}$ (dash-dotted), $E=15 \mathrm{mN} / \mathrm{m}$ (dashed),

499 and $E=30 \mathrm{mN} / \mathrm{m}$ (thin solid).

501 The elasticity of thin mineral oil film is poorly known, therefore, for estimation of

502 the oil film properties from the observed MSS contrasts, we propose to use the spectral

503 model (17) with the MSS defined by (19). Yellow, black and blue curves in Fig. 3 (right)

504 show simulations of the MSS contrasts caused by an oil film with the elasticity $E$, of 5 ,

50515 , and $30 \mathrm{mN} / \mathrm{m}$. Although the scattering of the data is strong, the model simulations

506 with $E=15 \mathrm{mN} / \mathrm{m}$ produce the best fit to the data. This value differs from $E=4 \mathrm{mN} / \mathrm{m}$

507 used in studies of oil slicks (Stanislav Ermakov, personal communication).

508 RIM simulations of the NRCS contrasts (C-band, VV, up-wind radar look direction 509 and incidence angle $30 \mathrm{deg}$ ) of the slicks caused by a thin oil film with $E=5,15$, and

$51030 \mathrm{mN} / \mathrm{m}$ are then shown in Fig. 10 (right). The NRCS contrasts are calculated for two

511 types of the scattering model: "pure" Bragg scattering model, the NRCS is ${ }^{\sigma_{0 b r}^{p}}$, and the 512 composite model accounting for the radio-wave scattering from breaking waves, the 
513 NRCS becomes $\sigma_{0}^{p p}=\sigma_{0 \mathrm{br}}^{p p}+\sigma_{0 \mathrm{~b}}$, where $\sigma_{0 b}$ represents the contribution by breaking

514 waves to radar backscatter. Further details are provided in Kudryavtsev et al. (2005). For

515 the first model, the NRCS contrasts correspond to the contrasts of wave spectrum on the

516 Bragg wavenumber, $k_{b r}$ (see spectra shown in Fig. $11 \mathrm{~b}$ on $k_{b r}=10^{2} \mathrm{rad} / \mathrm{m}$ ). For the other

517 model, the NRCS contrasts combine suppression of Bragg wave spectrum and wave

518 breaking. Following RIM, the range of breaking waves providing non-Bragg scattering is

519 defined as $k<k_{R} / 10$. For a C-band radar instrument, this is equivalent to wavelengths

$520>60 \mathrm{~cm}$. As follows from Fig. 11b, these waves are not affected by the film damping, and

521 the NRCS contrasts predicted by this model are lower than in the case of a "pure" Bragg

522 scattering model. If wave spectrum at $k=k_{b r}$ is significantly suppressed in the slick area

523 (as it is apparently seen in Fig. $11 \mathrm{~b}$ and in Fig. 10 (right) for the film with $E=15$ and

$52430 \mathrm{mN} / \mathrm{m}$ ), the Bragg scattering mechanism is switched off and the NRCS is mostly

525 supported by the breaking waves. In this case, the ratio of the NRCS between clean and

526 slick areas, $\sigma_{\text {Oclean }}^{p p} / \sigma_{\text {oslick }}^{p p}$, mostly corresponds to the inverse ratio of non-Bragg NRCS to

527 the total NRCS of the clean surface, i.e. $\sigma_{\text {oclean }}^{p p} / \sigma_{\text {oslick }}^{p p} \approx \sigma_{0}^{p p} / \sigma_{0 \mathrm{~b}}$.

528 The averaged NRCS contrast for the oil slick in Fig. 9 (upper) is reported in Fig. 10

529 (right), as well as averaged NRCS contrasts obtained from ASAR of the same area on

530 May 25, 2010, 15:47 GMT. Experimental estimates of the NRCS contrast are consistent

531 with the model estimates for $E=15 \mathrm{mN} / \mathrm{m}$ and $E=30 \mathrm{mN} / \mathrm{m}$. Equality of the NRCS

532 contrasts results from the fact that wave damping coefficient at $k_{b r}=10^{2} \mathrm{rad} / \mathrm{m}$ for these

533 values of the elasticity turns to be the same (see Fig. 11a). Thus, in this case the model

534 NRCS contrasts fail to discriminate the surface slicks caused by films of different

535 elasticity. Referring to Fig. $11 \mathrm{~b}$ we may conclude that due to complicated shape of the 
536 spectral contrasts, the NRCS contrasts should be strongly dependent on "geometry" of

537 radar observations (radar wavelength, incidence angle, look-direction) that makes hardly

538 probable an univocal interpretation of radar observations of surface slicks of different

539 origin (biogenic, mineral oil etc). As opposed to radar, the MSS contrasts are dependent

540 on the spectral cutoff which is directly linked to the elasticity of the surface film, i.e. to

541 its origin. In this context, optical observations of surface slicks can provide a chance to

542 discriminate biogenic slicks (expected elasticity is $25-30 \mathrm{mN} / \mathrm{m}$ ) from mineral oil slicks

543 that (following our estimates) have elasticity about $15 \mathrm{mN} / \mathrm{m}$.

\section{4. Conclusion}

545 A new method for quantitative interpretation of the MSS spatial anomalies in sun

546 glitter imagery is proposed. The retrieval algorithm uses a transfer function that relates

547 the sun glitter brightness contrast to the MSS contrasts. The transfer function can be

548 determined either empirically from observed 2D shapes of the sun glitter brightness, or

549 theoretically if a model of probability density function for the sea surface slope is known

550 and specified.

551 The method is applied to different sets of MODIS and MERIS sun glitter images. It is

552 shown that use of the two different methods for the analysis of coincident MERIS and

553 MODIS images gives very similar fields of the roughness anomalies. We further found

554 areas where the thickness of the oil films is significantly larger than the red light

555 wavelength. In these areas, the retrieved MSS anomalies are not realistic, even becoming

556 "bright," contradicting the expected suppression of short scale roughness in slicks. In

557 such cases, the optical properties of the oil itself (its "color") dominate, which have not

558 been taken into account in the method. 
In the area where oil film thickness is presumably thin, relative to the red light

560 wavelength, the oil slicks in the field of the MSS are visible as distinct dark features

561 (suppression of the MSS). We found contrasts of mineral oil slicks somehow lower than

562 the contrasts of biogenic slicks reported by Cox and Munk (1954). The different

563 elasticity of the crude and fish oils can explain this result.

564 The Radar Imaging Model (RIM) developed by Kudryavtsev et al. (2005) was

565 extended to simulate the optical images and further used to quantify this effect. As

566 estimated, the effective elasticity coefficient for the thin oil film is $E=15 \mathrm{mN} / \mathrm{m}$, with

567 model contrasts become consistent with observations.

568 Corresponding ASAR images provided an opportunity to assess similarities and

569 differences between the optical and radar signatures of the same oil spills. Except for the

570 area covered by thick (relative to red light wavelength) oil film, optical and radar

571 contrasts of the same slick are very well correlated. The NRCS changes in the oil slick

572 are stronger than the MSS ones. This is amplified at low wind speed with the radar

573 contrast significantly stronger than the optically-derived anomalies. RIM simulations of

574 SAR signatures, using a value of the oil film elasticity of $E=15 \mathrm{mN} / \mathrm{m}$, also provides

575 reasonable correspondence between model estimates and observations.

576 The method clearly provides new opportunities for quantitative investigations of

577 surface signatures of ocean phenomena, including internal waves and mesoscale ocean

578 currents. The roughness changes can indeed help in tracking and quantifying the surface

579 signatures of upper ocean motions. Interestingly, MSS changes can be quantified, and

580 synergy between SAR and sun glitter imagery can lead to a better understanding of the

581 manifestations of surface ocean phenomena. 
584 under the contracts №P1677, №02.740.11.5225 and №14.740.11.0201

\section{References}

Adamo, M., G. De Carolis, V. De Pasquale, and G. Pasquariello (2005), Combined use of SAR and MODIS imagery to detect marine oil spills, SAR Image Analysis, Modeling, and Techniques VII. Edited by Posa, Francesco, Proceedings of the SPIE, Volume 5980, pp. 153-164.

Apel, J. R., H. M. Byrne, J. R. Proni, and R. L. Charnell (1975), Observation of oceanic internal and surface waves from the Earth Resources Technology Satellite, $J$. Geophys. Res., 80, 865-881

Artale, V., D. Levi, S. Marullo, and R. Santoleri (1990), Analysis of Nonlinear Internal Waves Observed by Landsat Thematic Mapper, J. Geophys. Res., 95(C9), $16,065-16,073$.

Bolshakov, A. N., V.M. Burdyugov,, S.A.Grodsky, , V.N. Kudryavtsev, , a nd V.G. Proschenko, (1990a), Spectra of energy-carrying surface-waves using solar highlight images - comparison with in-situ data, Earth Obs. Remote Sens., 8, 2940.

Bolshakov, A.N. V.M. Burdjugov, .., S.A. Grodsky, and V.N. Kudryavtsev(1990b), 2dimensional surface elevation spectra from airphoto data, Izvestia, Atmos. Oceanic Phys., 26, 652-658. 
604 Bréon, F. M., and N. Henriot (2006), Spaceborne observations of ocean glint reflectance and modeling of wave slope distributions, J. Geophys. Res. 111, C06005, doi:10.1029/2005JC003343.

607

608

609

610

611

612

613

614

615

616

617

618

Burdyugov, V.M., S.A. Grodsky, and V.N. Kudryavtsev (1987), Analysis of photographic images of the structure of the surface of the sea near a speck of light, Physical Oceanography, 1, 55 - 62, 1987, DOI: 10.1007/BF02198288.

Chapron, B., V. Kerbaol, D. Vandemark, and T. Elfouhaily (2000), Importance of peakedness in sea surface slope measurements and applications, J. Geophys. Res., $105,17,195-17,202$.

Chust, G. and Y. Sagarminaga (2007), The multi-angle view of MISR detects oil slicks under sun glitter conditions, Remote Sens. Environ., 107, 1 -2, 232-239, doi:10.1016/j.rse.2006.09.024

Cox, C., and W. Munk (1954): Measurement of the roughness of the sea surface from photographs of the Sun's glitter. J. Opt. Soc. Amer., 44, 838-850.

Hennings, I., J. Matthews, and M. Metzner (1994), Sun glitter radiance and radar crosssection modulations of the sea bed, J. Geophys. Res., 99(C8), 16,303-16,326.

Hu, C., X. Li, W. G. Pichel, and F. E. Muller-Karger (2009), Detection of natural oil slicks in the NW Gulf of Mexico using MODIS imagery, Geophys. Res. Lett., 36, L01604, doi:10.1029/2008GL036119.

Jackson, C. (2007), Internal wave detection using the Moderate Resolution Imaging Spectroradiometer (MODIS), J. Geophys. Res., 112, C11012, doi:10.1029/2007JC004220. 
626 Jackson C.R., and W. Alpers (2010), The role of the critical angle in brightness reversals on sunglint images of the sea surface, J.Geoph. Res. VOL. 115, C 09019, doi:10.1029/2009JC006037

629 Johannessen, J.A., V. Kudryavtsev, D. Akimov, T. Eldevik, N. Winther, and B. Chapron 630 (2005), On Radar Imaging of Current Features; Part 2: Mesoscale Eddy and Current Front detection. J. Geoph. Res., 110, C07017.

632

634

635 636

Kudryavtsev V., D.Akimov, J.A.Johannessen, and B. Chapron (2005), On radar imaging of current features. Part 1: Model and comparison with observations, J.Geophys. Res. 110, C07017.

Levich, V. G. (1962), Physiochemical Hydrodynamics, Prentice-Hall, Upper Saddle River, N. J.

Matthews, J. P. (2005), Stereo observation of lakes and coastal zones using ASTER imagery. Remote Sens. Environ., 99, 1-2, 16-30, doi:10.1016/j.rse.2005.04.029.

Mitnik, L., W. Alpers, K. S. Chen, and A.J. Chen (2000), Manifestation of internal solitary waves on ERS SAR and SPOT images: Similarities and differences, Proceedings of the 2000 I nternational Geoscience and $R$ emote Sensing Symposium (IGARSS'00), Hawaii, USA, 24-28 July, Vol. 5, 1857-1859

Munk, W., P. Scully-Power, and F. Zachariasen (1987), Ships from Space, Proc. R. Soc., Ser. A, 412, 231- 254.

Munk, W., L. Armi, K. Fischer, and F. Zachariasen (2000), Spirals on the Sea, Proc. R. Soc., Ser. A, 456, 1217-1280. 
647 Stilwell, D., Directional energy spectra of the sea from photographs, J. Geophys. Res., $648 \quad 74,1974-1986,1969$

649 Vandemark D., et al., Ocean Wave Slope Observations Using Radar Backscatter and 650 Laser Altimeters, J. Phys. Ocean., 34, 2825-2842, 2004 